\title{
Major sepsis study confirms back to basics approach
}

\author{
D Nathwani \\ Professor and Consultant in Infectious Diseases, NHS Tayside, UK
}

TITLE A randomized trial of protocol-based care for early septic shock AUTHORS The ProCESS Investigators

JOURNAL N Engl J Med 2014; 370:1683-93. http://dx.doi.org//0.1056/ NEJMoa 1401602

DECLARATION OF INTERESTS No conflicts of interest declared.
Correspondence to D Nathwani, Infection \& Immunodeficiency Unit Ward 42, East Block Ninewells Hospital Dundee DDI 9SY, UK

tel +44 (0) I382 660 III

e-mail dilip.nathwani@nhs.net

\section{SUMMARY}

In 200I Rivers et al. published a landmark study in the New England Journal of Medicine showing that in a single centre, involving patients presenting to an emergency department with severe sepsis and septic shock, an early goal-directed therapy (EGDT) protocol significantly reduced mortality compared with those receiving usual care.' This underpinned the long-standing tenet of medical practice that early detection and treatment of sepsis will reduce mortality.

The recent ProCESS US randomised multi-centre study ${ }^{2}$ had the aim of determining whether the Rivers et al. findings were generalisable and whether all aspects of the protocol in relation to EGDT were necessary. The study identified some key findings: There was no difference in mortality (in-hospital mortality to 60 days, 90 day or one-year mortality) between the three arms. This may suggest a negative trial. However, overall mortality was significantly lower than reported in the Rivers study in 200I, although the ProCESS study also included very sick patients.

Levels of adherence in relation to early recognition and early antibiotic treatment were high and on a par with what one would realistically expect to achieve in real global practice. Therefore, ProCESS is a refining trial showing that early recognition and resuscitation are key beneficial interventions.

\section{REFERENCE}

I Rivers E, Nguyen B, Havstad S et al. Early goal-directed therapy in the treatment of severe sepsis and septic shock. $N$ Engl J Med 200 I; 345:I 368-77. http://dx.doi.org/I0.I056/NEJMoa0I0307

\section{COMMENTARY}

Sepsis is an important cause of morbidity and mortality. In Scotland there is a national drive, the Scottish Patient Safety Programme, to reduce sepsis-related death by promoting core quality improvement interventions. Therefore, new evidence to inform current practice is of significance.

I would suggest that the prompts and improved processes of care provided by the protocols improved early recognition, timely treatment and monitoring, thus driving the lower mortality observed. Clearly, since 200I, the paradigm shift advocated by Rivers et al. has moved our basic management to improve care. The lack of difference in the three arms (protocol-based EGDT, standard therapy protocols or usual care) suggests that no individual resuscitative path is worse or better, thereby allowing clinicians the flexibility to design the best local approach.

Furthermore, the requirement for intense invasive haemodynamic monitoring, which can be potentially harmful, is probably unnecessary; more focus on less costly, lower-risk alternatives such as lactate measurements are equally effective.

In summary, more technical care is not always better; simple interventions applied consistently and timeously are equally effective. We continue to streamline the effective interventions for improved sepsis management.

2 ProCESS investigators. A randomized trial of protocol-based care for early septic shock. N Engl J Med 2014; 370:1683-93. http:// dx.doi.org/I0.1056/NEJMoa I40I602 